\title{
Human Resource Management im Wandel der Digitalisierung
}

\section{Perspektiven cloudbasierter Human Resource Shared Services für kleine und mittlere Unternehmen}

Immanuel Zurbriggen und Jochen Schellinger

\section{Zusammenfassung}

In Zeiten der Digitalisierung sind die Unternehmen gezwungen, sich mit der Thematik übergreifend und funktionsbezogen auseinanderzusetzen. Große Unternehmen gelten durch ihre starke Ressourcenbasis als Vorreiter der Digitalisierung, während KMU hier tendenziell einen weniger weiten Entwicklungsstand haben. Auch das Human Resource Management (HRM) wird stark durch die Digitalisierung und die mit ihr verbundenen digitalen Transformation geprägt und muss seine Rolle im Unternehmen neu definieren. Die administrativen HR-Prozesse, die heute häufig noch von HRMitarbeitenden durchgeführt werden, werden zukünftig voraussichtlich durch die Mitarbeitenden selber oder durch voll automatisierte intelligenzbasierte und vernetzte HR-Systeme und Roboter abgewickelt. Auf Grundlage einer theoretischen Analyse wird ein für kleine und mittlere Unternehmen (KMU) einsetzbares mehrwertgenerierendes Modell für cloudbasierte HR Shared Services entwickelt, das im Rahmen einer qualitativen empirischen Untersuchung bei 13 Schweizer KMU und Großunternehmen gestaltungsbezogen validiert und optimiert wird.

I. Zurbriggen

Saas-Almagell, Schweiz

J. Schellinger $(\square)$

Berner Fachhochschule Wirtschaft, Bern, Schweiz

E-Mail: jochen.schellinger@bfh.ch 


\subsection{Einleitung}

Die Digitalisierung ist seit Jahren ein populäres Thema der wirtschaftswissenschaftlichen, technologie- und wirtschaftspolitischen Diskussionen. Was zunächst mit einem technologischen Fokus begann, ist längst in einer großen Breite in den Medien, der Politik und in Unternehmen und anderen Organisationen angekommen (Bauer und Hofmann 2018, S. 1). Die neuen Trendthemen heißen auch für das Human Resource Management (HRM) in vielen Unternehmen „,digitale Transformation“, „Cloud-Nutzung“ oder „Arbeit 4.0“. Insbesondere größere Unternehmen gelten durch ihre starke Ressourcenbasis als Vorreiter in der Digitalisierung, da sie die mit ihr verbundenen Vorteile besser nutzen und sich dadurch gegebenenfalls einen wettbewerbsseitigen Vorsprung verschaffen können. Vor diesem Hintergrund stellt sich die Frage, ob und inwiefern es in der Regel ressourcenschwächeren kleinen und mittleren Unternehmen (KMU) gelingt, mit dem technischen Fortschritt und dem mit ihm einhergehenden schnellen Wandel mitzuhalten, die zugehörigen Chancen zu nutzen und die resultierenden Herausforderungen zu bewältigen. Der nachfolgende Beitrag geht dieser Fragestellung nach und fokussiert dabei auf den Funktionsbereich HRM. Ein großes Potenzial für KMU wird hierbei in cloudbasierten Shared-ServicesAnsätzen vermutet. Auf der Grundlage einer theoretisch-konzeptionellen Bestandsaufnahme wird deshalb ein entsprechendes gestaltungsorientiertes Shared-Services-CloudModell entwickelt, das im Rahmen eines qualitativen Forschungsansatzes überprüft und optimiert wird. Im Kern soll folgenden Hauptforschungsfragen nachgegangen werden:

1. Welche Bedeutung kommt grundsätzlich dem Megatrend Digitalisierung für die Unternehmenswelt zu?

2. Welche Auswirkungen hat die Digitalisierung auf das HRM und inwiefern führt sie zu grundlegenden Veränderungen im HRM?

3. Wo stehen kleine, mittlere und große Schweizer Unternehmen bei der HRDigitalisierung?

4. In welche Richtung verändert sich das HRM voraussichtlich aufgrund der Digitalisierung in den nächsten fünf und mehr Jahren?

Außerdem stellen sich in Bezug auf die Nutzungsoption cloudbasierter Shared Services ergänzend noch folgende weiteren Fragen:

5. Welche Komponenten muss ein cloudbasiertes Shared-Services-Modell von der KMU-Bedarfsseite aus abdecken?

6. Welche Komponenten muss ein cloudbasiertes Shared-Services-Modell von der KMU-Angebotsseite aus abdecken?

7. Was sind wesentliche Aspekte cloudbasierter HR Shared Services für ein KMU?

Die Ergebnisse der Analyse sollen praxisrelevante Handlungsperspektiven für KMU durch ein cloudbasiertes HR-Shared-Services-Modell für das HRM aufzeigen, damit diese 
auch, trotz begrenzter Ressourcenverfügbarkeit, an den digitalen Zukunftsoptionen teilhaben können.

Für die Beantwortung der Forschungsfragen wird methodisch sequenziell vorgegangen. Zunächst werden die theoretisch-konzeptionellen Grundlagen auf Basis der verfügbaren Literatur analysiert und dann im entwickelten Modell integrativ zusammengeführt. Die Ergebnisse der konzeptionellen Analyse werden durch die eigene empirische Erhebung weiter fundiert und das Modell überarbeitet. Anschließend erfolgt eine Reflexion und perspektivische Hinterfragung.

\subsection{Konzeptionelle Grundlagen}

In Verbindung mit den allgegenwärtigen technischen Veränderungen unserer Berufs- und Arbeitswelt werden eine Vielzahl von Begriffen in den Raum gestellt, die auch für das HRM der Zukunft von großer Bedeutung zu sein scheinen. Häufig genannt werden insbesondere die Begriffe „Digitalisierung“, „Cloud“, „Künstliche Intelligenz“, „Big Data“, „Internet der Dinge“, „Industrie 4.0“ oder „Arbeitswelt 4.0“. Die zur Beantwortung der Forschungsfragen zentralen Begrifflichkeiten werden nachfolgend inhaltlich abgegrenzt und im Modell für cloudbasierte Shared Services im HRM zusammengeführt.

\subsubsection{Digitalisierung}

Zöller (2019) führt in Bezug auf die Relevanz der Digitalisierung für die Unternehmenswelt pointiert folgende Aussage an: „Wie wir es auch drehen und wenden, unsere Welt wird immer digitaler, und gleichzeitig nimmt die Bedeutung analoger Technologien stetig ab. So hart es für manchen klingen mag: Sich der Digitalisierung zu verschließen kommt einem Kampf mit Holzkeulen gleich, während die Gegner vollautomatische Waffen benutzen." Digitalisierung bezeichnet dabei einen technischen Prozess, bei welchem analoge Daten oder Abläufe in digitale umgewandelt werden. Die ganzheitliche Betrachtung des damit einhergehenden umfassenden Changeprozesses, der sowohl die Wirtschaft wie auch die Gesellschaft als Ganzes betrifft, wird als digitale Transformation bezeichnet (Peter 2017, S. 9). Digitalisierung schließt nach Ternès (2018, S. 1) auch die Automatisierung, Robotisierung, Virtualisierung und vor allem die Vernetzung mit ein. Digitalisierung basiert wesentlich auf den cyber-physischen Systemen (CPS), die eine Prozessverknüpfung zwischen Realität und virtuellen Daten ermöglichen und durch intelligente Software gesteuert werden (Drupp 2018, S. 70). Im ökonomischen Kontext bedeutet Digitalisierung konkret, Prozesse mit möglichst umfassender Unterstützung durch Software umzusetzen. Die definierten Tätigkeiten erledigen dann weitgehend die Computer oder Maschinen, während menschliche Eingriffe, soweit möglich und sinnvoll, reduziert werden (Fleischmann et al. 2018, S. 206). Im Unterschied zu früheren Technologiesprüngen gibt es in Verbindung mit der Digitalisierung viele Tätigkeiten, die ersatzlos wegfallen können, 
sodass bisherige Berufsbilder und Tätigkeitsprofile verschwinden und die dahinterstehenden Menschen sich neu am Arbeitsmarkt ausrichten müssen. Die Industrie 4.0 befindet sich mit der Digitalisierung der Fertigungsprozesse auf einem Weg, bei dem manuelle Tätigkeiten durch den Menschen in wachsendem Ausmaß ganz oder teilweise überflüssig gemacht werden, indem Arbeitsschritte und auch zu fertigende Geräte durch Software ersetzt werden. Für die Gesellschaft stellt sich in diesem Zusammenhang gesamthaft für die Zukunft die Frage, wie digitalisierungsbedingt freigesetzte Arbeitnehmende wieder sinnvoll am Arbeitsmarkt integriert werden können (Sendler 2018, S. 187 f.).

Wichtigster Treiber der digitalen Transformation sind die neuen Kundenbedürfnisse. Die Kunden sind zunehmend sehr gut informiert und vernetzt, verlangen maßgeschneiderte Lösungen sowie individuelle Produkte zu einem Preis von Massenware, erwarten eine bequeme und schnelle Lieferung und vorzüglichen Service. Nur diejenigen Unternehmen, die diesen Ansprüchen genügen und sich an den Kunden ausrichten, bleiben auf Dauer wettbewerbsfähig (Fleischmann et al. 2018, S. 10). In Bezug auf die internen Prozesse geht es bei der Digitalisierung wettbewerbsbezogen vor allem um die Erreichung einer optimalen Effizienz zur Kostensenkung und für Qualitätsverbesserungen bei Produkten und Dienstleistungen. Eine gelungene digitale Transformation wird insofern für viele Unternehmen in Zukunft zu einer Überlebensfrage werden (Zöller 2019, S. 12), die neue Kompetenzen und Fachwissen erforderlich macht (Zöller 2019, S. 41), die intern aufgebaut und extern akquiriert werden müssen. Das Finden und Fördern richtiger Talente und Fähigkeiten, die Erhöhung der Veränderungsgeschwindigkeit oder eine Veränderung der Unternehmenskultur in Richtung Flexibilität und Anpassungsfähigkeit, und damit wesentliche Aufgabenfelder des HRM, werden dabei immer wichtiger (Reinnarth et al. 2018, S. 253).

\subsubsection{Shared Services und Shared Service Center}

Die Bezeichnung „Shared“ im Terminus „Shared Services“ verweist darauf, dass eine Dienstleistung von mehreren Organisationseinheiten in Anspruch genommen wird (Brühl et al. 2017, S. 9 f.). Shared Services umschreibt in diesem Sinne eine Organisation von Verwaltungs- und Unterstützungsfunktionen auf einer professionellen Plattform in einem abgegrenzten organisatorischen, eigenverantwortlichen Bereich mit eigener Ergebnisverantwortung. Die Adressaten dieser Serviceeinheiten können sowohl interne wie auch externe Kunden sein. Das Ziel von Shared Services ist es, Unterstützungsdienstleistungen im Hinblick auf die Kosten, Qualität, Durchlauf- und Prozesszeit als wettbewerbsfähige Alternative zu Outsourcing-Lösungen anbieten zu können (Dressler 2007, S. 25). In diesem Zusammenhang ist es wichtig, die Unterstützungsaktivitäten der Shared Services von den Kernaktivitäten eines Unternehmens abzugrenzen, was sich angesichts der Dynamik der Wettbewerbsumfelder oftmals nicht einfach gestaltet. In Shared-Services-Konzepten spiegelt sich der Grundansatz von Shared Service Centern (SCC) wider, wonach Sekundärdienstleistungen durch Konzentration und Verlagerung in eine selbstständige Einheit zu einer Kernkompetenz dieser Einheit werden können. Im Vordergrund stehen dabei Effizi- 
enzgewinne sowie Qualitätssteigerungen durch verbesserte und synergetische Prozesse, die sich in Form von Economies of Scale niederschlagen, die transaktions- und volumengetrieben sind. Durch den Aufbau einer selbstständigen Organisationseinheit werden in ihr die Verantwortung für die betroffenen Prozesse gebündelt und in einem erweiternden Schritt die Möglichkeit geschaffen, die Dienstleistungen des SSC auch gegenüber Dritten zu vermarkten. Dadurch kann ein SSC zu einem eigenständigen Geschäftsbereich mit Gewinnverantwortung werden, der durch seinen Wertbeitrag eine Relevanz für den Unternehmenserfolg aufweist (Brühl et al. 2017, S. 19 f.). Ein Shared Service Center kann nach Moll (2012, S. 22), stellvertretend für eine Reihe ähnlich gelagerter Definitionen, wie folgt gekennzeichnet werden: „Shared Service Center sind rechtlich, organisatorisch oder wirtschaftlich selbstständige Verantwortungsbereiche, in denen vormals überwiegend dezentral erbrachte Unterstützungsprozesse konsolidiert und mehreren, vornehmlich internen Kunden als marktorientierte Dienstleistungen zur Verfügung gestellt werden. Ziel einer solchen Shared Services Architektur ist die Wertsteigerung des Gesamtunternehmens, was bspw. durch Effizienzverbesserungen oder Erhöhung der Prozess- und Servicequalität erreicht werden kann“. Unter Shared Services sind demnach gebündelte Dienstleistungen zu verstehen, die mit dem Ziel von Effizienz- und Effektivitätssteigerungen für mehrere interne und externe Kunden erbracht werden und die in einer separaten Organisationseinheit - in einem SSC - zusammengefasst werden (Brühl et al. 2017, S. 16).

Neben den standardisierten einfacheren Prozessen können durch die Digitalisierung auch komplexere Prozesse oder Dienstleistungen effizienter erbracht werden (Reinnarth et al. 2018, S. 29 f.). Dies hat einen direkten Einfluss auf SSC, die sich üblicherweise vor allem auf repetitive Aufgaben mit geringeren Kompetenzanforderungen an die Mitarbeitenden fokussieren. Die interne Wertschöpfung eines SSC basiert in der Regel auf einem hohen Automatisierungs- und Digitalisierungsgrad, einhergehend mit einem umfassenden Einsatz von Informationstechnologien (IT). Dies führt dazu, dass die Prozesse oder Teilprozesse eines SSC im Prinzip auch alternativ von externen Zulieferern oder spezialisierten Dienstleistern bezogen werden könnten. Die Kunden eines externen Servicedienstleisters erhielten in dem Fall beispielsweise die erbrachten Leistungen in digitaler Form, indem die Prozesse in einem ERP-System durch das externe SSC bearbeitet werden (Gleich et al. 2017, S. 152). In diesem Kontext stellt sich also die strategische Frage nach dem In- oder Outsourcing von digitalen Prozessdienstleistungen. Die technischen Voraussetzungen für beide Varianten sind durch die Digitalisierung vermehrt gegeben. Hoch spezialisierte Dienstleistungen sind ressourcenbedingt im Normalfall nur eingeschränkt auf Personenebene bündelbar. Bei zunehmendem, z. B. wachstumsbedingtem Bedarf ist vor einer Abteilungsbildung zu prüfen, ob diese nicht durch alternative externe Serviceoptionen entfallen kann. Im Zusammenhang mit der Digitalisierung werden insbesondere für Unternehmen mit Ressourcenengpässen solche Outsourcing-Überlegungen mit dem Ziel langfristig partnerschaftlicher Verbindungen mit entsprechenden Dienstleistungsanbietern vermehrt interessant. Im Idealfall besitzt ein solcher Partner die benötigten Ressourcen, kann sich auf die Gegebenheiten des Kundenunternehmens anpassen und wächst mit diesem mit (Reinnarth et al. 2018, S. 130 ff.). 


\subsubsection{Cloud und Cloud-Services}

In der Literatur existieren verschiedene Definitionen und Erklärungsansätze von Cloud Computing (Krcmar et al. 2018, S. 8 f.). Die Definition des National Institute of Standards and Technology (NIST), einer Bundesbehörde der Vereinigten Staaten, hat sich in der Fachwelt aber zwischenzeitlich als Grundlage etabliert. Nach NIST bezeichnet Cloud Computing ein Modell, ,... welches einen flexiblen und bedarfsorientierten Zugriff auf einen gemeinsam genutzten Pool von konfigurierbaren IT-Ressourcen ermöglicht, die jederzeit und überall über das Internet oder ein Netzwerk abgerufen werden können“ (zit. n. Mell und Grance 2011). Dies schließt beispielsweise den Zugriff auf Netzwerke, Server, Speicher oder Anwendungen mit ein. Mit einem möglichst minimalen Managementaufwand und geringer Interaktion werden Cloud-Services von einem Cloud-Service-Provider schnell und möglichst automatisch auf Basis von individuellen Kundenbedarfen an den Cloud-Service-Kunden angepasst und für ihn bereitgestellt. Damit die Sicherheit der Businessanwendung gegeben ist, verschlüsselt der Anbieter die Zugriffe mit einer anbieterunabhängigen Cloud-Verschlüsselung, wobei die Schlüssel beim Anwenderunternehmen verbleiben. Weiter verfügen Cloud-Anwendungen über ausgeklügelte Abwehrsysteme und es ist Aufgabe des Providers, fortlaufend präventiv an der Sicherheitsarchitektur zum Schutz der Kundensysteme zu arbeiten (Abolhassan 2016, S. 23).

Die Anforderung, im Extremfall Milliarden Devices weltweit miteinander stabil und zuverlässig zu vernetzen, erfordert eine Technologie, die Tempo und Skalierbarkeit gleichermaßen erfüllen kann. Cloud Computing hat sich hier als zukunftsfähige Lösung herauskristallisiert. Es bildet quasi das Rückgrat und die Intelligenz der gesamten Digitalisierung, denn Cloud Computing sorgt für mehr Speicher- und Datenanalysekapazität. Die dadurch gewonnen riesigen Datenmengen (Big Data) lassen sich unabhängig von der Userzahl in Echtzeit zentral sammeln und auswerten. Die Digitalisierung führt zu einer substanziellen Veränderung der Wertschöpfungsketten in allen Branchen und die Technologie der Cloud spielt dabei als Enabler eine entscheidende erfolgskritische Rolle. Mithilfe von skalierbaren, hoch verfügbaren und flexiblen Cloud-Lösungen sind die Unternehmen immer mehr in der Lage, so effizient wie noch nie auf Anforderungen des Marktes zu reagieren. Die aus der Verlagerung von Prozessen in die Cloud resultierende Flexibilität und Agilität muss aber simultan mit der Gewährleistung einer hohen Stabilität und Sicherheit des IT-Kernbetriebs realisierbar sein, um bestehende Wertschöpfungsprozesse nicht zu gefährden (Abolhassan 2016, S. 15-20).

„Cloud-Services“ bieten Unternehmen zahlreiche Möglichkeiten zu Kostenersparnissen. Insbesondere mittelständische Unternehmen können von den Vorteilen der CloudServices profitieren (Krcmar et al. 2018, S. 1). Gemäß dem Cloud-Monitor vom Jahr 2018 (KPMG 2018, S. 5) setzen bereits $66 \%$ der Unternehmen in Deutschland auf Cloud Computing und nur für $13 \%$ ist die Cloud nach wie vor kein Thema. Krcmar et al. (2018, S. 5) unterscheiden zwischen drei grundlegenden Servicemodellen (vgl. auch Schneider und Sunyaev 2015; Mell and Grance 2011):

- Software as a Service (SaaS): Der Cloud-Service-Kunde greift mit verschiedenen Endgeräten entweder über einen Thin-Client wie beispielsweise einem Web-Browser oder 
über ein Anwenderinterface auf die Softwareanwendungen zu. Die zugrunde liegende Cloud-Infrastruktur befindet sich unter der Kontrolle des Cloud-Service-Providers, während der Kunde nur spezifische Anwendereinstellungen vornehmen kann.

- Platform as a Service (PaaS): Der Cloud-Service-Kunde kann selbst entwickelte oder erworbene Anwendungen auf der Cloud-Infrastruktur des Cloud-Service-Providers installieren und betreiben. Wie beim SaaS hat der Kunde keine Kontrolle über die zugrunde liegende Cloud-Infrastruktur. Er kann aber gegebenenfalls eine limitierte Anzahl von Einstellungen in den eigens installierten oder ausgeführten Anwendungen vornehmen.

- Infrastructure as a Service (IaaS): In diesem Service-Modell erhält der Cloud-ServiceKunde Zugang zu den Hardwareressourcen des Cloud-Service-Providers, wie zum Beispiel auf die Rechenleistung, Speicherkapazitäten oder die Netzwerke. Der Kunde kann dabei beliebige Installationen von Software zum Betrieb verwenden, wie etwa Betriebssysteme oder Anwendungen. Diese werden vom Cloud-Service-Kunden kontrolliert, nicht aber die zugrunde liegende Cloud-Infrastruktur.

Soll ein solches Cloud-Service-Modell in einem Unternehmen zum Einsatz kommen, muss es skalierbar, einfach, sicher und bezahlbar sein sowie der Compliance des Kunden entsprechen. Weiterhin sind die Abläufe, Rollen und die Service-Levels eng zwischen Anbieter und Kunde abzustimmen. Die Auswahl eines geeigneten Partners mit passender Expertise und passendem Technologieportfolio ist entscheidend, um einen optimalen unternehmensspezifischen Cloud-Service zu erhalten, und dabei auch von zusätzlichen Kompetenzen in den Bereichen IT-Innovation, Transformation und Datacenter-Lösungen zu profitieren. Der Provider agiert dabei im Regelfall kundenbedarfsorientiert als zentraler Dreh- und Angelpunkt beim Projekt- und Qualitätsmanagement und adressiert das gesamte Wertschöpfungsnetzwerk. Viele Kunden wünschen sich gleichzeitig ein einfaches, intuitiv bedienbares Frontend, welches aber auch höchste Sicherheitsstandards im Backend erfüllt. Der Erfolg einer Cloud-Lösungen zeichnet sich dadurch aus, dass ein Cloud-Anbieter bei maximaler Kosteneffizienz in bestehende Umgebungen integrierbar ist und Unternehmens- und IT-Prozesse der Kunden nahtlos aufeinander abstimmen kann (Abolhassan 2016, S. 21 f.).

\subsubsection{Möglichkeiten von Human Resource Shared Services}

Im Zusammenhang mit der Digitalisierung nimmt das Human Resource Management (HRM) eine besondere Rolle ein, da es in zweierlei Hinsicht von der Digitalisierung betroffen ist. Zum einen werden die HR-Funktion und sämtliche Bereiche der HRWertschöpfungskette durch die Digitalisierung beeinflusst. Zum anderen ist das HRM durch die Veränderungen in anderen Unternehmensbereichen gefordert, das Unternehmen bei der Entwicklung geeigneter digitaler Kompetenzen zu unterstützen, um die digitale Transformation voranzutreiben (Konrad et al. 2018, S. 2). Der HR-Bereich entwickelt sich schon seit längerem und durch die mit der Digitalisierung verbundenen 
Automatisierungsmöglichkeiten verstärkt weg von einer Verwaltungseinheit hin zu einem internen Service- und Beratungsanbieter, der die Geschäftsbereiche bei ihrer Wertschöpfung unterstützt. Die Hauptaufgabe des HRM besteht demnach in Zukunft in der Mitwirkung an der Wertsteigerung des gesamten Unternehmens (Ternès 2018, S. 6-9). Besondere Bedeutung haben dabei zukünftig die Förderung von Kompetenzen für Prozesse und Veränderungen sowie die Mitwirkung bei der Lösung abstrakter und komplexer Probleme, um die Herausforderungen der digitalen Transformation bewältigen zu können. Die Digitalisierung steht ferner als Transformation eines soziotechnischen Systems mit den Komponenten Technik, Mensch und Organisation unmittelbar in Verbindung mit dem Tätigkeitsfeld des HRM, das Gestaltungsaufgaben in Bezug auf Menschen und Organisation verantwortet. Das HRM spielt insofern eine wichtige Rolle bei den strategischen Entscheidungen zur Digitalisierung, da passende Personalressourcen und stimmige Organisationsstrukturen für den Erfolg der Digitalisierung eines Unternehmens maßgeblich sind. Das HRM ist für die soziale und strukturelle Architektur der digitalen Transformation mit verantwortlich und fördert die Unterstützung der Mitarbeitenden bei der Umsetzung des digitalen Wandels (Armutat et al. 2018, S. 63-64). Die hohe Bedeutung von Führungs-, Struktur- und Prozessoptimierungsfragen der Digitalisierung für das HRM zeigt sich auch in den Ergebnissen der Kienbaum Trendstudie (2015). Im Rahmen der Erhebung wurden 187 Personalverantwortliche im deutschen Sprachraum befragt, welche HR-Handlungsfelder aufgrund der voranschreitenden Digitalisierung besonders an Bedeutung gewinnen. $65 \%$ der Befragten haben dabei die Führung und das Kulturmanagement genannt, $59 \%$ die Organisationsentwicklung und das Transformation Management und $48 \%$ die HR-Prozesse und das Systemmanagement der internen Digitalisierung (vgl. dazu Abb. 10.1).

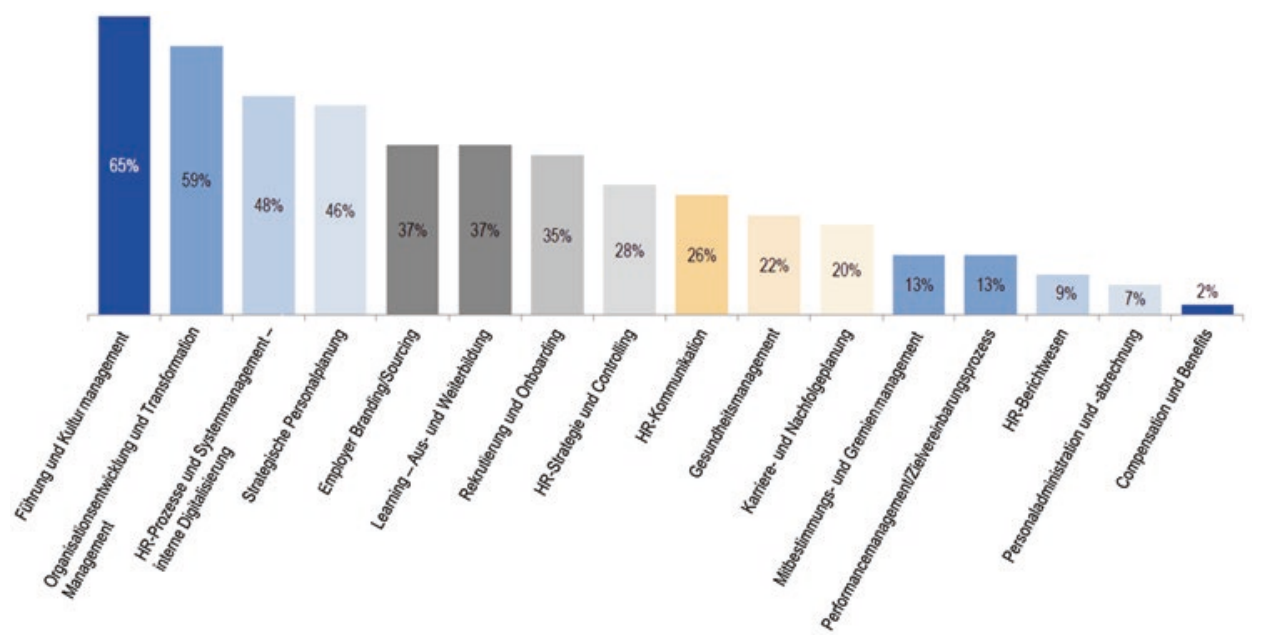

Abb. 10.1 Human-Resource-Handlungsfelder mit steigender Bedeutung aufgrund der Digitalisierung. (Quelle: Kienbaum 2015) 
Bei der Erfüllung der Aufgaben des HRM kommen aus Sicht der Führungskräfte auch im Zeitalter der Digitalisierung immer noch zwei Hauptanforderungen zum Tragen (Blume 2006, S. 68-77): Das HRM muss einerseits durch höhere Prozesseffektivität einen höheren Beitrag zur Wertschöpfung des Unternehmens leisten (,die richtigen Dinge tun“) und andererseits seine Leistungen effizienter erbringen, um die Unternehmenskosten durch Prozesseffizienz zu senken (,die Dinge richtig tun“). Nach Blume (2006, S. 68 ff.) können im Rahmen eines Professionalisierungs- und Entwicklungsprozesses mehrere HRM-Reifegrade identifiziert werden. Die erste Stufe beschreibt die klassische HRMFunktion mit reiner Supportaufgabe. Das HRM nimmt hier primär die Rolle als interner Dienstleister gegenüber der Geschäftsführung, den Führungskräften und den Mitarbeitenden wahr. Die zweite Reifegradstufe ist durch die Erstellung eines transparenten HRLeistungsangebotsportfolios mit definierten „HR-Produkten“ gekennzeichnet. Das HRM wandelt sich in dieser zweiten Stufe vom administrativen Experten zum kundenorientierten Dienstleister. In der dritten Reifegradstufe definiert der HR-Bereich nicht mehr einseitig den Umfang sowie die Güte der zu erbringenden HR-Dienstleistungen, sondern vereinbart schriftlich mit seinen Kunden ein Service Level Agreement (SLA) mit Kennzahlen zur Leistungsmessung, den sogenannten „Key Performance Indicators“ (KPI). Damit resultiert eine objektiv messbare Kunden-Lieferanten-Beziehung. Auf Stufe vier der Reifegradbetrachtung wird ein Shared Services Center aufgebaut, in dem dezentrale Ressourcen gebündelt und dadurch Skaleneffekte erzielt werden. Dies steigert die Prozesseffizienz, die Qualität der Personalarbeit sowie den zentralen Aufbau von IT- und HR-ProzessKnow-how. Die letzte Entwicklungsstufe ist durch ein Business Process Outsourcing (BPO) geprägt, bei welchem die Verantwortung und Durchführung einzelner oder mehrere HR-Prozesse an einen externen Dienstleister übertragen wird und der verbliebene interne HR-Bereich sich primär mit strategischen Aufgaben auseinandersetzt.

Eine ähnliche Perspektive auf ein zukunftsgerichtetes und hoch entwickeltes HRM zeigt Altmeier (2017, S. 10 ff.) im Drei-Säulen-Modell auf. Es umfasst zunächst die drei HR-Teilbereiche HR-Funktion, HR-Businesspartner und Center of Expertise, die jeweils durch spezifische Kompetenzen und Rollen gekennzeichnet sind. Darüber hinaus gibt es Lösungsansätze für das HRM, die die Betonung noch stärker auf Shared Services Center oder ein Business Process Outsourcing legen und in denen die klassische Personalabteilung teilweise aufgelöst wird, oder eine alternative Form der Leistungserbringung durch die Einführung eines strategisch ausgerichteten HR Service Delivery Models realisiert wird. Nahezu alle Zukunftsmodelle des HRM weisen neben einer strategischen Beratungs- und Dienstleistungsfunktion auch eine Komponente zur integrierten und effizienten Erbringung von spezialisierten administrativen HR-Dienstleistungen auf. Je nach Ausgestaltung kann diese als HR Shared Services Center (HRSSC) oder auch als HR Shared Services (HRSS) ausgewiesen werden. Ziel einer solchen internen und gegebenenfalls auch externen Integration ist die Optimierung von Kosten, Durchlauf- und Prozesszeiten. Das vorrangige Ziel sind allerdings zumeist die Kosten, die durch Fixkostendegression, Kompetenzbündelung und eine höhere Effizienz durch Lernkurven der Mitarbeitenden gesenkt werden sollen. Gleichzeitig wird durch messbare Wertbeiträge die Kostentrans- 
parenz erhöht und ein höheres Kostenbewusstsein geschaffen. Zudem sollen eine stärkere Kundenorientierung und Service Level Agreements auch Qualitätsverbesserungen nach sich ziehen (Altmeier 2017, S. 16 f.). Ein Beispiel für die strukturelle Umsetzung eines zukunftsgerichteten Service-Delivery-Ansatzes im HRM findet sich bei Scherer (2017, S. 206). Sie beschreibt den sogenannten Future Mode of Operation (FMO) der E.ON Business $\mathrm{GmbH}$ und stellt die finale Reorganisation der HR-Prozesse, IT-Systeme und des Service Delivery Models grafisch dar (vgl. Abb. 10.2) dar. Der FMO vereint alle Prozesse rund um das Talentmanagement im Unternehmen und ist via Schnittstellen mit der Payroll und einem ESS/MSS-Portal verknüpft.

In technologischer Hinsicht bieten neue, insbesondere sogenannte On-Demand-basierte Softwarepakete, die als Cloud-Software oder Mietsoftware auf dem Markt erscheinen, erweiterte Möglichkeiten gegenüber den physischen Installationen von HR-Software im Unternehmen vor Ort (On-Premises). Diese Angebote eröffnen auch für den HR-Bereich effizientere und einfachere Standardisierungsmöglichkeiten mit einem noch höheren Kostensenkungspotenzial (Jakob 2017, S. 135).

Die voranschreitende Digitalisierung hat einen entscheidenden Einfluss auf die Handlungs- und Lösungsmöglichkeiten sowie Weiterentwicklungsstrategien für HR Shared Services Center. Themen wie Work-Life-Balance und die Erfordernis zur ständigen Erreichbarkeit, IT-Technologiesprünge und mobiles Cloud Computing sowie der wachsende Bedarf für Employee Self Services werden sich auch auf die Ausgestaltung von HRSSC auswirken (Mund 2017, S. 288 f.). Zur Cloud-Basierung von HR-Prozessen und HR Shared Services konnten in der gesichteten Literatur keine vertiefenden Beiträge identifiziert werden. Es finden sich diesbezüglich primär kundenorientierte Anmerkungen von

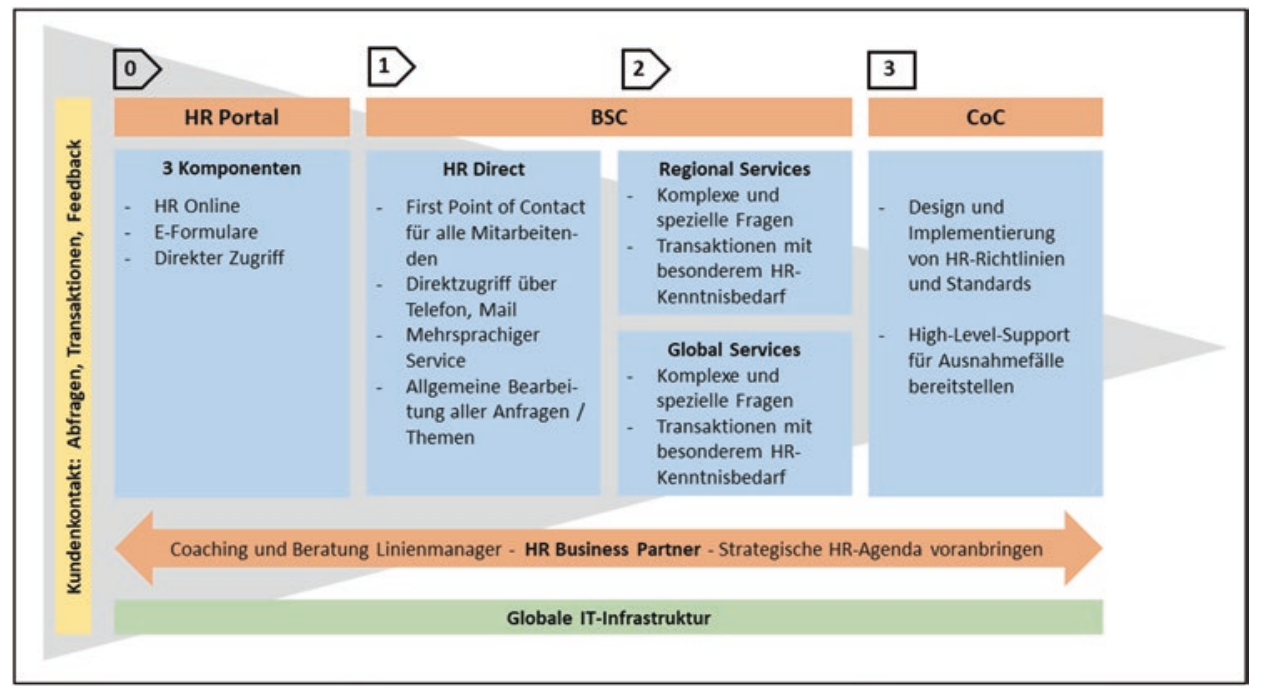

Abb. 10.2 Service Delivery Model (Quelle: Eigene Darstellung in Anlehnung an Scherer 2017, S. 216) 
HR-Softwareanbietern im Internet, die darauf verweisen, dass sie auch cloudbasierte Lösungen anbieten.

\subsubsection{Modellentwicklung}

Erfolgreiche Unternehmen definieren sich heute nicht mehr ausschließlich über ein Produkt oder eine Marke, sondern bieten ein Gesamtsystem an Produkten, Dienstleistungen und Vernetzungsmöglichkeiten an. Dabei ergeben sich unterschiedliche Leistungsbereiche, die kundenorientiert in Serviceoptionen miteinander verknüpft sind. Der Anbieter muss in diesem System eine hohe Innovationsgeschwindigkeit mit intelligenten, automatisierten Prozessen anbieten (Armutat et al. 2018, S. 109 f.). Zunehmend gefordert ist ein digitales, respektive digitalisiertes Geschäftsmodell, bei dem wesentliche wertschöpfende und betriebswirtschaftliche Faktoren durch den Einsatz von digitalen Technologien verbessert oder automatisiert werden und das kontinuierlich an die Veränderungen des wirtschaftlichen und technischen Umfelds angepasst wird (Greff et al. 2018, S. 58). Die digitale Transformation zwingt die Unternehmen, eine vielfach über die Jahre veraltete IT auf den neuesten Stand zu bringen. Das gilt auch für die HR-IT und die zugehörige HRSoftware. Oft muss dies im laufenden Betrieb geschehen, unter Sicherung höchster Qualitätsstandards und mit dem Einsatz von unterschiedlichen Expertinnen und Experten (Abolhassan 2016, S. 19 f.).

Der Kundenkontakt bzw. die Kundenkontaktpunkte sind für den Unternehmenserfolg besonders relevant. Das Ziel ist es, die Kommunikationskanäle inhaltlich-konzeptionell, prozessual-technologisch und kommunikativ so zu verbinden, dass den Kunden an allen Kontaktpunkten automatisiert und dynamisch ein Mehrwert geboten wird (Reinnarth et al. 2018, S. 113). In Bezug auf die internen und externen Kunden des HR-Bereichs umfasst die mögliche Palette, die durch einen externen HR-Dienstleistungspartner angeboten werden könnte, die Aufgabenfelder Payroll, Personaladministration, Bewerbermanagement, Stellenplanung, Corporate Identity, Outplacement, Abrechnung, Mitarbeiterbefragungen, Employer Branding, Personalmarketing, Reisemanagement bis hin zu Benefits und zum betrieblichen Gesundheitsmanagement (Armutat et al. 2018, S. 238).

Die Mitarbeitenden eines Unternehmens sind Leistungserbringer und betriebswirtschaftlicher Kostenfaktor in einem und zeichnen sich durch ständig verändernde Qualifikationen und wachsende Betriebserfahrung aus. Der effektive und effiziente Einsatz und die Weiterentwicklung der Humanressourcen wird in Zukunft, trotz oder gerade wegen der Digitalisierung, noch mehr an Bedeutung gewinnen. Dem muss der Personalbereich durch Struktur- und Prozessoptimierungen und eine stärkere Ausrichtung an den Bedürfnissen der Zielgruppen im HRM gerecht werden. Dies gilt auch für KMU, die ihr HRM effizienter und strategischer ausrichten müssen. Für viele KMU dürfte der erforderliche Veränderungsprozess sowohl quantitativ wie qualitativ schwer alleine realisierbar sein, sodass man von einem wachsenden Bedarf an externer professioneller Unterstützung ausgehen (Armutat et al. 2018, S. 231-232). Ein qualitativ hochwertiger Leistungsausbau muss mit einer 
verstärkten Serviceorientierung einhergehen, die durch Service Level Agreements erreicht werden kann, die an den HR-Kundenbedürfnissen ausgerichtet und durch die Definition von KPI gemessen werden (Altmeier 2017, S. 16-17).

Bei einer professionellen Begleitung von KMU-HR-Bereichen bei der Optimierung ihrer Strukturen und Prozesse sind ressourcenbedingte Restriktionen und Hemmnisse zu berücksichtigen, mit denen KMU sich dabei auseinandersetzen müssen (vgl. dazu C. Leyh et al. 2018, S. 31 ff.) und die im Falle einer partnerschaftlichen Zusammenarbeit von HR-Serviceanbieter und Unternehmen maßgeblich sind: Gewährleistung der IT-Sicherheit, keine hohen Investitionskosten, Rechtssicherheit, ein hohes Maß an Expertinnen- und Experten-Know-how sowie eine moderne IT-Infrastruktur. In Bezug auf diese Aspekte konnten Großunternehmen in der Vergangenheit einen Vorsprung bei der Digitalisierung des HRM gegenüber den mittelgroßen Unternehmen realisieren. KMU hingegen können dem mit größerer Handlungsschnelligkeit und Flexibilität bei der Entscheidungsfindung begegnen (Armutat et al. 2018, S. 230). Ausgehend vom angeführten FMO-Ansatz der E.ON (Scherer 2017) soll ein HR-Strukturmodell entwickelt werden, das versucht, die festgestellten Optimierungsrestriktionen beim HRM für KMU zu entschärfen und professionelle HR-Prozesse zu ermöglichen, die denen von Großunternehmen nicht nachstehen. Eine wesentliche Komponente dieses Ansatzes ist ein auf das HRM hin ausgerichtetes Grundverständnis von Shared Services (Moll 2012, S. 16), die in einer separaten Organisationseinheit, dem HRSSC, erbracht werden und zum Ziel haben, Effizienz- und Effektivitätssteigerung für Kundinnen und Kunden zu erbringen. Dabei sollen grundsätzlich Shared Services von einer internen Leistungseinheit oder alternativ auch von einem externen Partner im Auftragsverhältnis angeboten werden können, sodass ein KMU selber entscheiden kann, ob und wann es Teilaufgaben auslagern oder doch selbst ausführen will. Das Modell soll als virtuelle Private Cloud in Form eines Software-as-a-Service(SaaS)-Modells (Krcmar et al. 2018, S. 5-11) konzipiert werden, das gemeinsam mit einem externen Servicepartner realisiert wird. Zentrale Komponente ist ein cloudbasiertes HR Shared Services Center mit der Option, einzelne Teilbereiche ganz auslagern zu können (Outsourcing). Das Modell sieht auch einen bedarfsabhängigen fachlichen HR-Support durch das Partnerunternehmen vor. Die HR-Software wird im Modellansatz beim professionellen Partner gehostet und von diesem angeboten (SaaS), sodass die Aspekte der Datensicherheit, des Datenschutzes sowie die gesetzlichen Anforderungen (Compliance) jederzeit gewährleistet sind. Um dies sicherzustellen sollte bei der Zusammenarbeit auf eine Zertifizierung des Payroll-Bereichs von Swissdec (2019) geachtet werden, die den Lohnstandard der Schweiz sowohl für Quellsysteme (Payroll) wie auch die Übermittlung des Datensenders (Unternehmen) von Lohndaten definiert. Die nachfolgende Abb. 10.3 veranschaulicht die einzelnen Komponenten des Modells.

Das Modell bildet in der Grundstruktur vier Stufen der Servicenutzung ab und soll die Erbringung von HR-Dienstleistungen eines externen HR-Servicepartners gegenüber einem Kunden (KMU) darstellen. Durch den trichterförmigen Aufbau der Informationskaskade sollen Anfragen der Mitarbeitenden je Stufe bedarfsgerecht adressiert werden, sodass die Expertinnen und Experten nicht mit Anfragen konfrontiert werden, die auch auf 


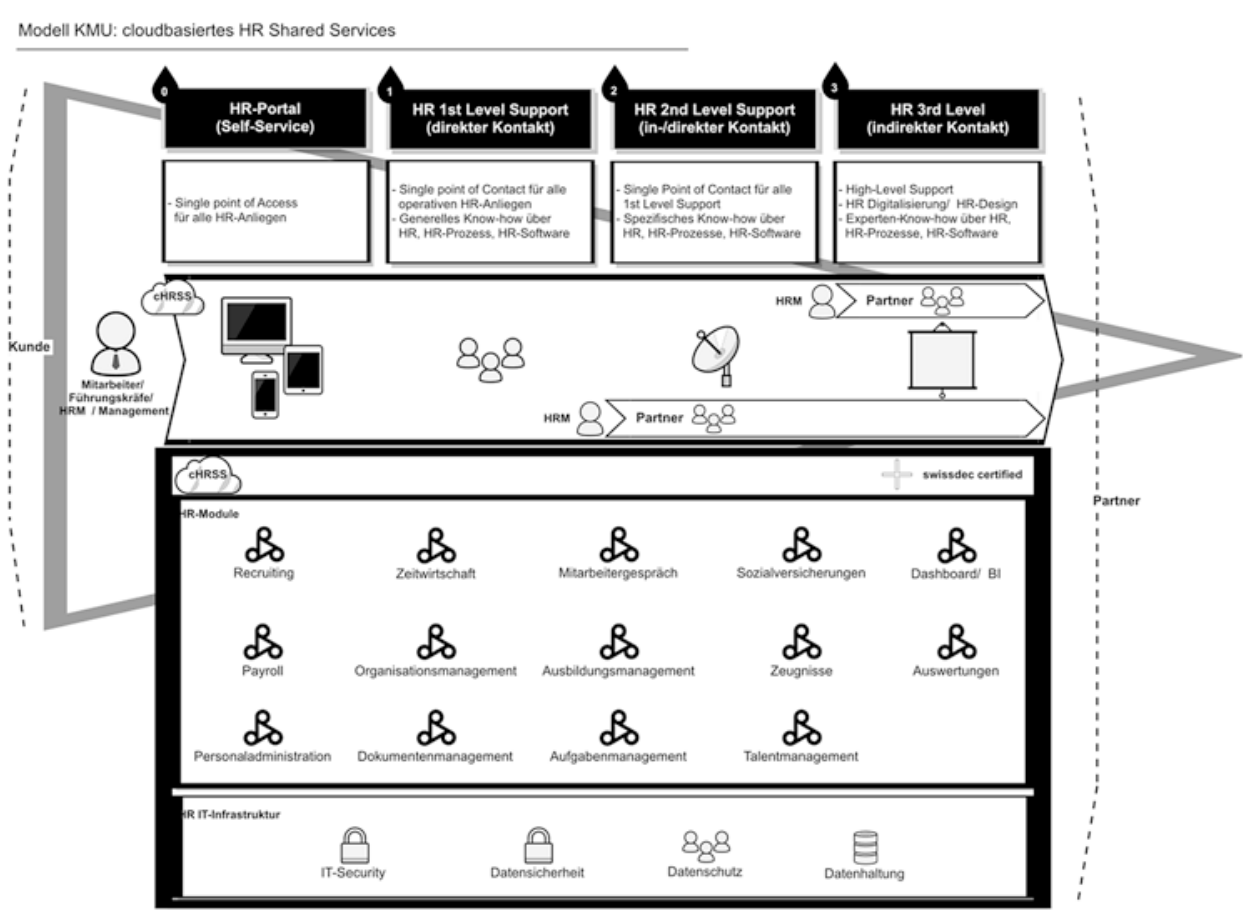

Abb. 10.3 Cloudbasierte Human Resource Shared Services für Klein- und mittelständische Unternehmen. (Quelle: eigene Darstellung)

einem niedrigeren Support-Level hätten beantwortet werden können. Die Mitarbeitenden, Führungskräfte sowie das HRM und Management des Kunden-KMU greifen in einer ersten Stufe (Stufe 0) auf das cloudbasierte HR-Portal zu und agieren hier eigenständig im Self-Service-Modus mit den entsprechenden Datenzugriffen oder dem Auslösen von verschiedenen automatisierten HR-Prozessen. Der Zugriff erfolgt dabei via Webbrowser oder über eine entsprechenden Mobile-Applikation für Smart Devices. Reicht dies nicht aus, dann können die Mitarbeitenden und Führungskräfte mit dem HR-Bereich resp. einem HR Support Center als First Level Support (Stufe 1) per E-Mail, via Telefon oder einem Ticketsystem Kontakt aufnehmen. Bedarf es Know-how eines Spezialisten im Second Level Support (Stufe 2) oder einer Expertin im High Level Support (Stufe 3), sind diese beziehbar. Die Fachpersonen der Stufen zwei und drei stehen in der Regel nicht im direkten Kontakt mit der Linie. Da KMU oft das nötige Spezialisten- und Experten-Know-how nicht selber aufbauen können oder wollen, erlaubt das Partnermodell hier, auf das Knowhow des Partners zurückzugreifen.

Das Partnermodell bietet gleichzeitig auch die Möglichkeit, HR-Software und die dafür benötigte Infrastruktur cloudbasiert zu beziehen (Hosting). Durch das flexibel aufgebaute modulare Angebot kann das KMU einerseits aus einem oder mehreren HR-Modulen resp. aus HR-Prozessen auswählen, die es einsetzen möchte und die funktional 
und technisch vollständig integriert sind. Die jeweiligen HR-Module werden vom Partnerunternehmen als „KMU-Standard“ ausgeliefert, damit die Kosten für ein KMU sich in Grenzen halten, individuelle Anpassungen sind aber mit Zusatzkosten prinzipiell möglich. In Bezug auf die HR-Module ist eine freie Wählbarkeit der operativen Abwicklung in Eigenregie oder im Rahmen des Partnervertrags möglich (z. B. vollständiges Outsourcing der Payroll-Abwicklung). Das Modell sieht unter Qualitäts- und Sicherheitsgesichtspunkten vor, dass das operative administrative HR-Geschäft in der Schweiz durchgeführt wird. Auch die Datenhaltung der HR-Software liegt in der Schweiz. Die dazu benötigte Infrastruktur wird dem Kunden als Hosting angeboten und durch Expertinnen und Experten geschützt (Cloud Security). Die Einhaltung der Datenschutz- und Datensicherheitsrichtlinien liegt in der Verantwortung des Partnerunternehmens. Das digitale Partnermodell heißt für das KMU, dass es bei sich weder Software installieren noch sich um das Releasemanagement kümmern muss. Die Software wird regelmäßig vom Partner aktualisiert und der Kunde entsprechend über Erweiterungen via Webinar oder Schulungen vor Ort informiert. Aus Sicht des Partnerunternehmens stellt das Partnermodell ein Geschäftsmodell dar, das zulässt, dass der Partner mehrere Kunden durch seine Angebotspalette bedienen kann, da die HR-Software mandantenfähig ist. Durch diesen Skaleneffekt und die oben beschriebenen Maßnahmen ergibt sich ein Business Case, bei dem alle beteiligten Parteien, insbesondere aber die KMU, profitieren. Als Kostenmodell wird ein On-Demand-Ansatz vorgeschlagen, das heißt, der Kunde bezahlt in einem Mietmodell und nur in dem Moment, in dem er eine HR-Software effektiv nutzt oder eine HR-Dienstleistung bezieht. Vor dem Hintergrund des hohen Kostenbewusstseins der KMU erscheinen einmalige Lizenzzahlungen als nicht zielführend. Qualität und Umfang der Leistungen werden über ein Service Level Agreement festgelegt, das flexibel gemäß den Kundenbedürfnissen im Zeitablauf angepasst werden kann. Das Modell repräsentiert somit ein Gesamtsystem aus HR-Produkten, HR-Dienstleistungen und Vernetzungsmöglichkeiten, das modular und flexibel aufgebaut ist und den KMU intelligente automatisierte HR-Prozesse ermöglicht, die dem Unternehmen eine Konzentration auf das Kerngeschäft erlauben und gegebenenfalls auch zur Kostensenkung im HR-Bereich beitragen.

\subsection{Empirische Untersuchung}

Im Rahmen der empirischen Untersuchung wurde analysiert, inwiefern sich die befragten Schweizer Unternehmen mit der Digitalisierungsthematik und Optionen für Shared Services auseinandersetzen und wie sie generell die zukünftige Entwicklung des HRM einschätzen. Die Aussagen und Einschätzungen wurden anschließend als Basis für eine kritische Bewertung und Neujustierung des entwickelten Partner- resp. Geschäftsmodells für eine cloudbasierte Shared-Services-Lösung für das HRM von KMU herangezogen. Nachfolgend werden Methodik und Vorgehen der empirischen Analyse beschrieben. 


\subsubsection{Forschungsmethode und -design}

Für das empirische Forschungssetting wurde ein qualitativer Ansatz mit halbstrukturierten leitfadengestützten Interviews mit Expertinnen und Experten von Schweizer KMU und Großunternehmen gewählt. Dies hat den Vorteil, dass die Thematik durch ein offenes aber systematisches Vorgehen mit großer Breite erfasst werden kann. Die Erhebung erfolgte in Anlehnung an die methodologischen Merkmale von Helfferich (2011, S. 334). Die Frageformulierung und die Reihenfolge der Fragestellung wurde in Abhängigkeit von der Interviewsituation flexibel angepasst, der Leitfaden wurde vorgängig nicht ausgehändigt und für alle Interviews wurde derselbe Interviewleitfaden verwendet. Während des Interviews wurde mit Rück- und Verständnisfragen interaktiv auf die Befragten eingegangen. Das Vorgehen erlaubt eine gründliche Analyse auf Basis von aufgezeichneten Texten und deren Interpretation. Die Textaufzeichnungen sind unverzerrt, authentisch und können intersubjektiv nachvollzogen und beliebig reproduziert werden (Lamnek und Krell 2016, S. 313). Der Interviewleitfaden orientiert sich an den sechs Grundprinzipien von Helfferich (2011, S. 334): Offenheit, begrenzte Fragenanzahl, formale Übersichtlichkeit, Orientierung am natürlichen Erinnerungs- und Argumentationsfluss, kein Ablesen von Fragen und Priorisierung von spontan produzierter Erzählung. Für die qualitative Datenerhebung wurdenprimärPersonenindenFunktionenHR-Leitung,HR-Digitalisierungsverantwortliche oder Mitglieder der Geschäftsleitung in den Unternehmen kontaktiert. Die 13 geführten Interviews fanden während drei Wochen im Frühling 2019 statt und wurden in jedem Fall vor Ort bei den Befragten in einem Sitzungszimmer durchgeführt. Insgesamt wurden über 588 Minuten Interviewmaterial gesammelt, geprüft, sortiert und subsumiert. Sämtliche Interviews wurden mit einem Diktiergerät aufgenommen. Die Tonspuren wurden anschließend jeweils in Dokumente (MS Word) wörtlich transkribiert. Dabei wurden Wortverschleifungen an das Schriftdeutsch angenähert sowie die Schweizer Dialekte in das Schriftdeutsch übersetzt. Gleichzeitig wurden die Satzform sowie der Satzinhalt beibehalten. Wort- und Satzabbrüche wurden geglättet bzw. weggelassen. Die Aufbereitung der transkribierten Daten für die qualitative Analyse erfolgte mit der Software ATLAS.ti auf Basis eines Codierschemas, das sich an die Struktur des Interviewleitfadens anlehnt. Die Codegruppen stehen dabei für die Themenbereiche und die Codes für die Fragen und zugehörigen Antworten aus dem Leitfaden. Diese wurden jeweils mit einer eindeutigen Identifikationsnummer versehen. Ferner wurde auf eine Zuordenbarkeit der Aussagen zu verschiedenen Unternehmensgrößen geachtet. Die Auswertungen der Codes erfolgten mittels Query Tool sowie der Code-Dokument-Tabelle in ATLAS.ti.

Eine möglichst hohe Objektivität der qualitativen Analyse sollte durch eine Berücksichtigung der sechs Gütekriterien von Mayring (2016, S.144 ff.) gewährleistet werden: Das Verfahren für die Ergebnisgewinnung ist genau dokumentiert und nachvollziehbar (Verfahrensdokumentation). Interpretationen sind nicht gesetzt, sondern argumentativ begründbar (argumentative Interpretationsabsicherung). Die Analyseschritte wurden vorgängig festgelegt und bedarfsabhängig angepasst (Regelgeleitetheit). Die Datenerhebung erfolgte im „Feld“, d. h. in den Unternehmen vor Ort (Nähe zum Gegenstand). Die 
Interviews wurden dialogisch und mit Nachfragen geführt (kommunikative Validierung). Durch Vergleich der Resultate mit vorhandenem Wissen und größenabhängige Hinterfragungen sollte die Qualität der Ergebnisableitung weiter verbessert werden (Triangulation).

In der qualitativen Sozialforschung wird eine bewusste Fallauswahl aus einer Grundgesamtheit getroffen, welche die Heterogenität des Untersuchungsfeldes falltypologisch repräsentiert. Für die Erhebung wurde auf eine maximale Variationsbreite geachtet, damit die Stichprobe aus unterschiedlichen Feldtypen aufgebaut ist (Clerc 2018, S. 36). Es wurden Unternehmen in neun unterschiedlichen Branchen und in vier Größenkategorien (klein, mittel, groß, groß 1000+) befragt. Die Stichprobe umfasst ein Kleinunternehmen bis 49 Mitarbeitende (7,7 \%), fünf mittelgroße Unternehmen bis 249 Mitarbeitende $(38,5 \%)$, drei Großunternehmen ab 250 Mitarbeitende $(21,3 \%)$ und vier Großunternehmen mit 1000 und mehr Mitarbeitenden (30,8 \%). In Tab. 10.1 ist ersichtlich, wie sich die Stichprobe der Interviewpartnerinnen und -partner konkret zusammensetzt.

\subsubsection{Ergebnisse}

\subsubsection{Digitalisierung und Human Resource Management}

Aus Sicht des Befragten im Kleinunternehmen (KU) sind von der Digitalisierung alle Geschäftsprozesse eines Unternehmens betroffen, die papierlos, schneller und effizienter abgewickelt werden können. Die Vertreterinnen und Vertreter der mittelgroßen Unternehmen (MU) sehen eine stufenweise Entwicklung in der Digitalisierung, die analoge Produkte zunehmend digital werden lässt und in einer Folgestufe generierte Daten zur Mehrwertgenerierung im Geschäftsmodell nutzbar macht. Sie verbinden mit der Digitalisierung eine flexible und unabhängig von Zeit und Raum mögliche Datennutzung, die mit erhöhten

Tab. 10.1 Sample

\begin{tabular}{l|l|l|l}
\hline Nr. & Funktion & Branche & Unternehmensgröße \\
\hline 1 & Leitung Marketing (Mitglied der GL) & Tourismus & Mittel \\
\hline 2 & Leitung HR & Versicherung & Mittel \\
\hline 3 & $\begin{array}{l}\text { HR-Fachspezialist und Verantwortung } \\
\text { Digitalisierung }\end{array}$ & Banken & Groß 1000+ \\
\hline 4 & Leitung HR & Handel & Mittel \\
\hline 5 & Chief Broking Officer (Mitglied der GL) & Versicherung & Groß \\
\hline 6 & Leitung HR & Versicherung & Groß \\
\hline 7 & Leitung HR & Pharma & Mittel \\
\hline 8 & Leitung HR & Medien & Mittel \\
\hline 9 & Leitung HR & Druck & Groß \\
\hline 10 & Leitung HR-Digitalisierung & Verkehr/Transport & Groß 1000+ \\
\hline 11 & Leitung HR & Banken & Klein \\
\hline 12 & Leitung HR-Digitalisierung & Telekommunikation & Groß 1000+ \\
\hline 13 & Leitung HR & Versicherung & Groß 1000+ \\
\hline
\end{tabular}

Quelle: eigene Darstellung 
Anforderungen zum Schutz vor Missbrauch einhergeht. Für die Befragten der Großunternehmen (GU) war eine Unterscheidung von Digitalisierung und Automatisierung insofern relevant, als Digitalisierung immer noch mit menschlichen Inputs einhergehe, während dies bei der Automatisierung im Prinzip nicht mehr der Fall sei. Digitalisierung stehe gleichzeitig auch für eine Grundhaltung und kundenorientierte Nutzung digital handelbarer Dienstleistungen. Die Befragungsteilnehmenden der großen Unternehmen mit über 1000 Mitarbeitenden (GU+) verbinden mit der Digitalisierung in einem ersten Schritt das Wegkommen von physischen Grenzen, die sich in einem zweiten Schritt in kommunikativen Systemvernetzungen niederschlagen und in einer dritten Stufe in einheitliche, standardisierte Tools und Prozesse münden, die Best-Practice-Problemlösungen ermöglichen. Es ginge dabei auch darum, die Geschäftsprozesse digital neu zu denken oder neue Geschäftsprozesse zu entwickeln, die nur durch die Digitalisierung möglich geworden sind, wie etwa durch Nutzung von künstlicher Intelligenz und Maschinenlernen in Datenkontexten. In Bezug auf die Mitarbeitenden gehe die Digitalisierung mit Aufgabenstellungen und neuen beruflichen Anforderungen einher, die intellektuell anspruchsvoller seien.

Der KU-Vertreter sieht die Digitalisierung auch im Kontext des HRM als relevant an und verweist beispielhaft auf einen automatisierten Spesenprozess, der via Mobile App abgewickelt werden kann oder Mutationsmeldungen an die Sozialversicherungen, die online getätigt werden können. Papier dürfte seiner Einschätzung nach auch in kleinen Unternehmen im HRM zukünftig verschwinden. Die MU-Befragten verbinden mit der Digitalisierung im HRM verstärkt den Einsatz von Cloud-Lösungen, die eine bessere Kommunikation und jederzeitige Datenzugriffe auf Personaldaten wie Mitarbeiterdossiers erlauben. Es gehe bei der Digitalisierung im HRM ferner darum, sämtliche administrativen Prozesse und das Recruiting digital abzubilden und dabei den Mitarbeitenden und Führungskräften mehr Verantwortung zu übertragen, etwa durch autonome Datenpflege und Anstoßen von Routineprozessen. Für die GU-Teilnehmenden heißt Digitalisierung des HRM eine vernetzte digitale Begleitung des gesamten Lebenszyklus von Mitarbeitenden und aller zugehörigen administrativen Prozesse wie Recruiting, Dokumentenmanagement, Eintritt, Payroll, Zeugniserstellung und Austritt. Die GU+-Befragten betonen in Verbindung mit der Digitalisierung des HRM die Notwendigkeit für eine neue Rollenklärung und geänderte kundennähere Aufgabenadressierungen. Gleichzeitig sollten in den Prozessen im HRM die regulatorischen Bedingungen technisch korrekt in den Systemen abgebildet sein und reibungslos funktionieren. HRM-Digitalisierung bedeute jedoch nicht nur, bestehende Prozesse digital abzubilden, sondern diese digital neu zu denken und, sofern möglich und sinnvoll, mit künstlicher Intelligenz anwenderfreundlich auszugestalten. Digitale HRM-Lösungen sollten möglichst schnell, intuitiv bedienbar und einfach sein. Dabei sei sicherzustellen, dass verwendete Technologien den erforderlichen Reifegrad hätten. In Bezug auf das Recruiting werden Automatisierungstools und Robotic-Lösungen favorisiert, die helfen, Profilinformationen zu sammeln, Skills zu identifizieren und potenzielle Mitarbeitende in Bezug auf Aufgaben- und Teamerfordernisse einschätzen zu können. Digitalisierung im Personalbereich wird aufgrund von Effizienzsteigerungen außerdem im Regelfall mit einer Reduzierung des Beschäftigungsvolumens in Verbindung 
gebracht, das es erlaubt, mit dem gegebenen Ressourcenbestand mehr oder anspruchsvollere Aufgaben zu übernehmen. Es gäbe aber Bereiche, die nicht oder nicht vollständig digitalisiert werden sollen oder können. Bestimmte HR-Aufgaben würden auch zukünftig nicht ohne die Einbindung von Menschen funktionieren, vor allem, wenn es um die umfassende Einschätzung von Personen und soziale Interaktionen gehe.

Insgesamt sehen die Befragten der Unternehmen in Bezug auf die Digitalisierung des HRM derzeit den Fokus auf die Themen Recruiting, Mitarbeitergespräche und Zweitwirtschaft sowie generell auf eine Prozessautomatisierung und -standardisierung in der Personaladministration gerichtet. Eine Erhöhung des Self-Service-Anteils und eine durchgängige Transparenz der Prozesslandschaft sei anzustreben. Bedenken werden gegenüber der Verfügbarkeit und Funktionsfähigkeit einer allmächtigen „All-in-one HR-Software“ geäußert, und dahingehend, dass der Fokus künftig zu stark auf Prozessperfektionierung und zu wenig auf die Kunden des HRM gelegt werde.

\subsubsection{Human Resource Shared Services}

Für den KU-Vertreter sind Shared Services ,geteilte Leistungen“ in dem Sinne, dass ein Kompetenzzentrum mit Fachspezialistinnen und -spezialisten administrative Tätigkeiten für andere ausführen oder sie darin unterstützen. MU-Befragte sehen in Shared Services zentralisierte Angebote, die intern und extern in Form von Dienstleistungen angeboten werden. Ihrer Ansicht nach setzt die Einrichtung von Shared Services eine gewisse Größe des Unternehmens voraus, damit die erforderlichen Bündelungen und Standardisierungen Sinn machen und kosteneffizient realisiert werden können. Die GU-Teilnehmenden kennzeichnen Shared Services als Zentralfunktion die definierte Dienstleistungen anbietet, über verschiedene Bereiche hinweg einheitlich gestaltet ist und dadurch Synergien bei repetitiven Aufgabenstellungen nutzen kann. Ähnlich sehen die GU+-Befragten Shared Services als Zusammenfassung, Standardisierung und Automatisierung aller administrativen Prozesse, bei der auch künstliche Intelligenz und entsprechende Algorithmen zum Einsatz kommen könnten. In dem Zusammenhang seien die Prinzipien von Effektivität und Effizienz zu beachten und würden sich Cloud-Lösungen anbieten. In Verbindung mit der Digitalisierung könne man auch kritisch hinterfragen, ob Shared Services nicht schon eine überholte Entwicklung seien, und die Digitalisierung bereits weiter gehe.

In Bezug auf das HRM kommen Shared Services beim KU primär für administrative, standardisierte und repetitive HR-Tätigkeiten wie Payroll, Mutationen und Jahresendverarbeitungsprozesse, die eher im „Hintergrund“ ablaufen, infrage. Auch für die mittelgroßen Unternehmen sind es jene HR-Dienstleistungen, die zentral, einheitlich und standardisiert erbringbar sind, die sich für HR Shared Services eignen. Die vorangehenden Grundkennzeichnungen des Begriffes werden entsprechend auf das HRM übertragen. Durch die unterstützenden Prozesse sollen die HR-Beratenden entlastet und die frei werdende Zeit für hochwertigere Aufgaben genutzt werden. Bei den großen Unternehmen werden HR Shared Services bereits operativ eingesetzt. Die Bandbreite reicht hier von einer zentralen internen Ausführung aller HR-Dienstleistungen bis zur externen Auslagerung von HR-Teilaufgaben. Auch die GU+-Unternehmen sehen in HR Shared Services 
(HRSS) eine schlanke Integration, Standardisierung und Harmonisierung aller administrativen HR-Prozesse. Für die Unternehmen mit mehr als 1000 Mitarbeitenden geht es bei HRSS darum, die gesamten administrativen HR-Prozesse in einer organisatorischen Einheit zusammenzufassen, zu standardisieren, zu harmonisieren und schlank und einheitlich anzubieten.

Die Befragten aller Unternehmen sehen den Mehrwert eines HRSS vorwiegend in der Standardisierung und Vereinheitlichung der HR-Prozesse, der resultierenden Professionalisierung des HRM und in den hiermit einhergehenden Kosten- und Ressourceneinsparungen, die die Effizienz des HRM erhöht und eine Konzentration auf das HR-Kerngeschäft erlaubt. Als Herausforderung bei einem HRSS wird die Sicherstellung der erforderlichen Fachkompetenz der Einheit, respektive eines eventuellen externen Partners gesehen. AuBerdem gehe durch die Bündelung der Prozessbearbeitung eventuell Know-how im Sinne eines übergreifenden Prozessverständnisses bei den HR-Mitarbeitenden verloren. Nicht zu vernachlässigen sei eine erforderliche Akzeptanz bei den Mitarbeitenden und Führungskräften, die vielfach Wert auf individuelle Betreuung aus einer Hand legten. Befürchtet wird ferner ein zusätzlicher Koordinationsaufwand zwischen dem HRM und dem HRSS, sofern dieses bei einem Partner ausgelagert wird.

\subsubsection{Cloud-Lösungen für das Human Resource Management}

Der KU-Befragte äußert sich eher skeptisch in Bezug auf die wenig greifbare und eventuell unsichere Nutzung von datensammelnden virtuellen Servern, die er mit dem CloudAnsatz in Verbindung bringt. Die MU-Befragten sehen in der Cloud technisch orientiert einen gemeinsamen externen Speicherplatz, in dem Daten transferiert und ausgetauscht werden, auf die ortsunabhängig jederzeit zugegriffen werden kann. Auch hier ist eine gewisse kritische Distanz zur Thematik wahrnehmbar, weil Unklarheiten hinsichtlich der Datenhoheit bestehen würden und teils widersprüchliche Einschätzungen auch bei ITFachexperten vorhanden seien. Wenn die erforderliche Daten- und Prozesssicherheit gegeben ist, sei aber eher unwichtig, wer technisch hinter der Cloud-Anwendung stehe. Bei den GU-Befragten werden Clouds als externe Datenplarkplätze betrachtet, bei denen sich die zentrale Frage stelle, wem die Daten gehörten. Clouds werden als weiterer Schritt in die virtuelle Zukunft gesehen, der Ressourceneffizienz in Verbindung mit Storage und dem Informationsaustausch verspricht. Auch die GU+-Befragten verweisen auf die externe, raumunabhängige Datenhaltung als zentrale Cloud-Eigenschaft. Zudem unterscheiden sie zwischen internen Private und externen Public Clouds, in denen die Daten irgendwo auf der Welt abgespeichert sind, durch entsprechende Verschlüsselungen aber nicht unbedingt unsicherer seien, als in einer Private Cloud. In technischer Hinsicht werden Clouds als Entwicklungstendenz der Informatik in Richtung Megarechenzentren gesehen, in denen Systemapplikationen prozessorientiert einfacher zusammengeführt werden. Dadurch würden Releasemanagement und Deployments massiv einfacher und skalierbarer und IT-Ressourcen effizienter einsetzbar.

Im Kontext des HRM sieht der KU-Vertreter die Cloud als eine webbasierte Toollösung, die viele Fragen zu Compliance, Datenschutz und Kundengeheimnissen aufwirft. 
Bei den MU wird die Cloud als Grundlage gesehen um HRSS zentralisiert anzubieten und den Zugriff auf HR-Daten und -Prozesse jederzeit und ortsunabhängig zu gewährleisten. Als bekanntes Beispiel hierfür wurde ein cloudbasiertes Recruiting-Tool genannt, welches als Standardmodul bezogen und extern gehostet werden kann und somit nicht mehr in house auf eigenen Servern installiert werden muss. Bei den großen Unternehmen sind bei cloudbasierten HRM-Lösungen sowohl der Datenschutz wie auch die Datensicherheit wichtige Punkte, die in Abstimmung mit dem Partner spezifiziert werden müssen. Die Befragten der sehr großen Unternehmen sehen Clouds im HRM vor allem im SaaS-Kontext als sinnvolle Perspektive. Die Datensicherheit in der Cloud wird als weniger kritisch betrachtet, da es inzwischen technische Möglichkeiten gebe, diese zu gewährleisten. Es wird auch auf Bestrebungen der IT-Verantwortlichen hingewiesen, die möglichst alle Themen unternehmensweit in die Cloud transferieren möchten, wovon das HRM nicht ausgeschlossen sei. Die mit Cloud-Angeboten oftmals artikulierten Ängste wurden als weniger technisch begründet, sondern vielmehr politisch-emotional bedingt angesehen.

Alle Unternehmen sehen den Mehrwert von HR-Cloud-Lösungen in potenziellen Ressourcen- und Kosteneinsparungen, die insbesondere auf das externe Hosting, das Releasemanagement sowie die Nutzung von externem IT-Know-how zurückzuführen sind. Zentrale standardisierte Cloud-Lösungen und der Einsatz von Best-Practice-Anwendungsmodulen, die jederzeit up to date sind, sind weitere Argumente für deren Einsatz. Aus Sicht der Anwender der HR-Systeme ist die jederzeitige und ortsunabhängige Verfügbarkeit von Prozessen und Daten hervorzuheben. Es werden von allen Befragten auch Möglichkeiten zur Auslagerung von HRM-Risiken gesehen, die etwa bei einem Ausfall von internen Single-Know-how-Trägern gegeben sind. Essenziell für eine Nutzung von Cloud-Angeboten im datentechnisch sensiblen HRM-Umfeld ist allerdings eine hinreichende Vertrauensbasis in Bezug auf die Serviceanbieter und Datenschutz, Datensicherheit und Prozesssicherheit. Diesbezüglich wurde fast durchgängig der Anspruch geäußert, dass sowohl die operative Abwicklung wie auch das Hosting in der Schweiz lokalisiert sein sollten.

\subsubsection{Modell für cloudbasierte Human Resource Shared Services}

Die Teilnehmenden der Untersuchung wurden in den Interviews auch nach möglichen Inhalten und Komponenten eines cloudbasierten HR-Shared-Services-Modells befragt. Ein entsprechendes Modell sollte gemäß dem Gros der Befragten vor allem den Aspekt „Payroll“ abdecken und eine digitale Unterstützung oder Abnahme aller administrativen HR-Tätigkeiten über den ganzen Mitarbeitenden-Life-Cycle sicherstellen. Spezifische Unterstützungsbedarfe in den Bereichen Mitarbeitergespräche, Recruiting und Zeitwirtschaft wurden hierbei betont. Weitere HR-Dienstleistungen wie das Ausbildungsmanagement, Krankheits- und Unfallmeldungen sowie Arbeitsrechtsberatung wurden ebenfalls als wünschenswert angeführt.

Ein Kriterium, das cloudbasierte HRSS gemäß der Befragten zwingend erfüllen muss, sind Effizienzsteigerungen durch schnellere und effizientere HR-Prozesse und, damit verbunden, Ressourceneinsparungen. Gleichzeitig spielt das Preis-Leistungs-Verhältnis eine 
wesentliche Rolle sowie die Verfügbarkeit eines verlässlichen und vertrauensvollen Partners, der persönliche Kontakte pflegt. Die offerierten cloudbasierten Lösungen müssen einfach und benutzerfreundlich sein, die IT-Security und die Compliance muss jederzeit gewährleistet und eine hohe Verfügbarkeit sowie die Datenhaltung in der Schweiz garantiert sein.

Cloudbasierte HRSS sollten als übergeordneter Mehrwert eine Freiraumschaffung für das HR-Kerngeschäft und insgesamt eine Professionalisierung des HRM realisieren. Cloudbasierte HRSS mit einem externen Partner scheinen sich aufgrund der Vorteile beim Ressourceneinsatz, der technologischen Aktualität und IT- und Datensicherheit, der Professionalisierung durch Standardisierung und der Verfügbarmachung von zusätzlichem Fach-Know-how im besonderen Maße für KMU zu eignen. Kritische Aspekte der Einführung von cloudbasierten HRSS sind die gegebenenfalls sehr hohe Abhängigkeit von einem externen Partner und die Externalisierung bisher intern vorhandenen HR-Fachwissens. Eine Kontrollierbarkeit der Aktivitäten des Partners ist im Fall von Nichteinhaltung von vereinbarten Standards, etwa bei der IT- und Datensicherheit, nur bedingt gegeben. Außerdem wird durch die Prozessstandardisierung gegebenenfalls die interne Flexibilität und personenbezogene Servicequalität beeinträchtigt.

\subsubsection{Zukünftige Entwicklungen im Human Resource Management}

Summarisch über alle Teilnehmenden der Befragung hinweg wurden eine Reihe von vermuteten Entwicklungstrends für das HRM der Zukunft angeführt. Eine deutliche Reduzierung administrativer Tätigkeiten auf ein Minimum scheint im HR-Bereich für die Zukunft wahrscheinlich: Alles, was regelbasiert, repetitiv oder von gesetzlichen Bestimmungen her fix definiert ist, kann und wird wohl auch standardisiert und voll automatisiert werden. Denkbar wäre in diesem Kontext eine deutliche Reduzierung der Präsenz des HR-Bereichs in vielen Teilgebieten bis hin zu einer völligen Eliminierung von HR-Abteilungen im traditionellen Sinne, die mit einer vermehrten Übernahme von personalbezogenen Entscheidungsbedarfen in der Linienführung einhergeht. Eine wichtige Rolle wird hier der künstlichen Intelligenz beigemessen. Als bereits verfügbare Beispiele werden Avatare angeführt, die Erstgespräche mit Bewerbenden führen sowie Algorithmen, die passende Kandidatinnen und Kandidaten selbst finden und vorschlagen und in einem extremen Szenario die Einstellungsentscheidung sogar eigenständig vornehmen und bis zur Vertragsgestaltung abwickeln.

Trotz der Digitalisierung wird allerdings aus Sicht der Befragten im Personalbereich auch in Zukunft der Mensch eine wichtige Rolle spielen. Die Rolle von HR-Mitarbeitenden wird sich aber voraussichtlich stark verändern. Diese werden vermehrt als SparringPartnerinnen und -Partner der Linie agieren, diese intensiver begleiten und betreuen und sich mit mitarbeiterbezogenen Fragen der digitalen Transformation als Change Agents auseinandersetzen müssen. Der HR-Bereich wird voraussichtlich ein immer wichtiger werdender Treiber von Kultur- und Organisationsentwicklung und ein Befähiger der Mitarbeitenden zur Sicherstellung des zukünftig erforderlichen Skill-Sets. Personalmitarbeitende werden zukünftig deshalb auch über veränderte Kompetenzprofile verfügen müssen. 
Neben psychologischer Grundkenntnisse und Empathie wird voraussichtlich zukünftig verstärkt ein Verständnis der betriebswirtschaftlichen Kerngeschäfte und Zusammenhänge gefordert sein, wozu auch digitale Kompetenzen notwendig sein werden. Komplexere Themen wie die Laufbahnplanung, das Talentmanagement oder die Führungskräfteentwickung werden für den Alltag künftig vermutlich prägender. In Verbindung mit der Digitalisierung werden auch weiterhin neue Arbeitsmodelle entstehen. Als Beispiel mit hoher Zukunftsrelevanz wird die „Gig Economy“ angeführt, die für das HRM die vermehrte temporäre projektbezogene Akquise von Mitarbeitenden oder ganzer Teams zur Folge haben wird, die das Unternehmen nach Aufgabenerledigung wieder verlassen. Der zunehmende Wettbewerbsdruck dürfte vor allem auch bei KMU dazu führen, dass eine noch weitergehendere Konzentration auf wertschöpfende Prozesse erfolgen muss und der Druck zum Outsourcing von HRM-Teilfunktionen oder aller HR-Aktivitäten steigt. Zu Beginn dürften zunächst alle administrativen Tätigkeiten auf deren Externalisierbarkeit geprüft werden, zuvorderst die Lohn- und Gehaltsabrechnung.

\subsubsection{Synthese und Konsequenzen}

Aus der summarischen Komprimierung der empirischen Ergebnisse gehen größenabhängig teils unterschiedliche Einschätzungen der Relevanz von Teilaspekten einer Digitalisierung des HRM hervor, die mit unterschiedlichen digitalen Reifegraden der befragten Unternehmen erklärbar sind. Während Großunternehmen im Gegensatz zu KMU etwa digitale Lösungen für Feedbackprozesse und das Ausbildungsmanagement präferieren, sehen KMU eher Anwendungen für die Zeitwirtschaft oder das Recruiting als drängender. Großunternehmen fokussieren vor allem auf eine umfassende Prozessautomatisierung, während KMU prozessbezogen eher auf priorisierte Einzelaspekte abheben. Die zentrale Relevanz der Prozessoptimierung wird vor allem von den mittelgroßen Unternehmen betont, die hier das derzeit größte Potenzial zur Mehrwertgenerierung sehen. Dies schlägt sich auch in deren Bewertung der Potenziale von HRSS nieder, die mit großen Chancen für eine Effizienzsteigerung und Professionalisierung des HRM und der erforderlichen Konzentration auf das Kerngeschäft in Verbindung gebracht werden. Dies entspricht auch den Erkenntnissen der vorangehenden theoretisch-konzeptionellen Analyse, wonach vor allem mittelgroße Unternehmen bei der HRM-Digitalisierung einen Aufholbedarf im Vergleich zu Großunternehmen aufweisen. So sind Großunternehmen im Gegensatz zu den KMU größtenteils im HRM bereits stark automatisiert und wenden sich bereits recht konkret Zukunftsthemen wie der digitalen Identität, künstlicher Intelligenz oder Robotik zu. Der größenabhängig unterschiedliche Reifegrad der Digitalisierung im HRM hat sich bei den Interviews auch bei der Bewertung von begriffsgeprägten Teilaspekten der Digitalisierung gezeigt, die mit zunehmender Unternehmensgröße auf größere Erfahrungshintergründe und fachliche Vertiefungen schließen ließen.

Das entwickelte Modell für cloudbasierte HRSS ist bei den befragten Personen größenunabhängig auf ein positives Echo gestoßen. Insbesondere aber die KMU haben in diesem Zusammenhang zum Ausdruck gebracht, dass Bedarf für eine Unterstützung bei der HRM-Digitalisierung besteht und die Befragten sehen ein großes Potenzial für ein ent- 
sprechendes HRSS-Partner- resp. Geschäftsmodell. Das vorgestellte cloudbasierte HRSSModell wurde von den Befragten als zukunftsfähig und praktikabel eingestuft. Unsicherheiten bei der Modellpositionierung gab es seitens der Großunternehmen hinsichtlich des Umfangs der enthaltenen Outsourcing-Aspekte. Das Modell ist diesbezüglich von der Grundidee her offen und sehr flexibel ausgerichtet und deckt an sich eine Bandbreite von „ganz" bis „gar nicht“ ab. Durch geringfügige Anpassungen in der Modelldarstellung und eine Umbenennung zu „HR as a Service“ (HRaaS) soll dies stärker hervorgehoben werden. Deutlicher werden soll dadurch auch, dass seitens eines (Kunden-)Unternehmens sowohl HR-Dienstleistungen, als auch HR-Software bezogen werden können. Das empiriebasiert leicht modifizierte Modell findet sich in Abb. 10.4.

Die veränderte Darstellung hebt ferner grafisch die Bedeutung der Datenhaltung in der Schweiz und die „,24/7“-Verfügbarkeit der Angebote hervor. Die Module wurden aufgrund der Aussagen neu unterteilt in die Kategorien „HR-Basis“ (sämtliche administrativen Tätigkeiten) und „HR-Module“ (weitergehendere HR-Prozessanwendungen). Die HRBasiskategorie wurde noch um die Thematik ,Krankheits- und Unfallmeldungen“ ergänzt. Im Hinblick auf die HR-IT-Infrastruktur erfolgt eine Erweiterung um den Aspekt der Cloud Security, und es wurde vor dem Hintergrund der geforderten großen Offenheit des Systems eine Ergänzung um Schnittstellen vorgenommen, die die Anbindung in- oder ex-

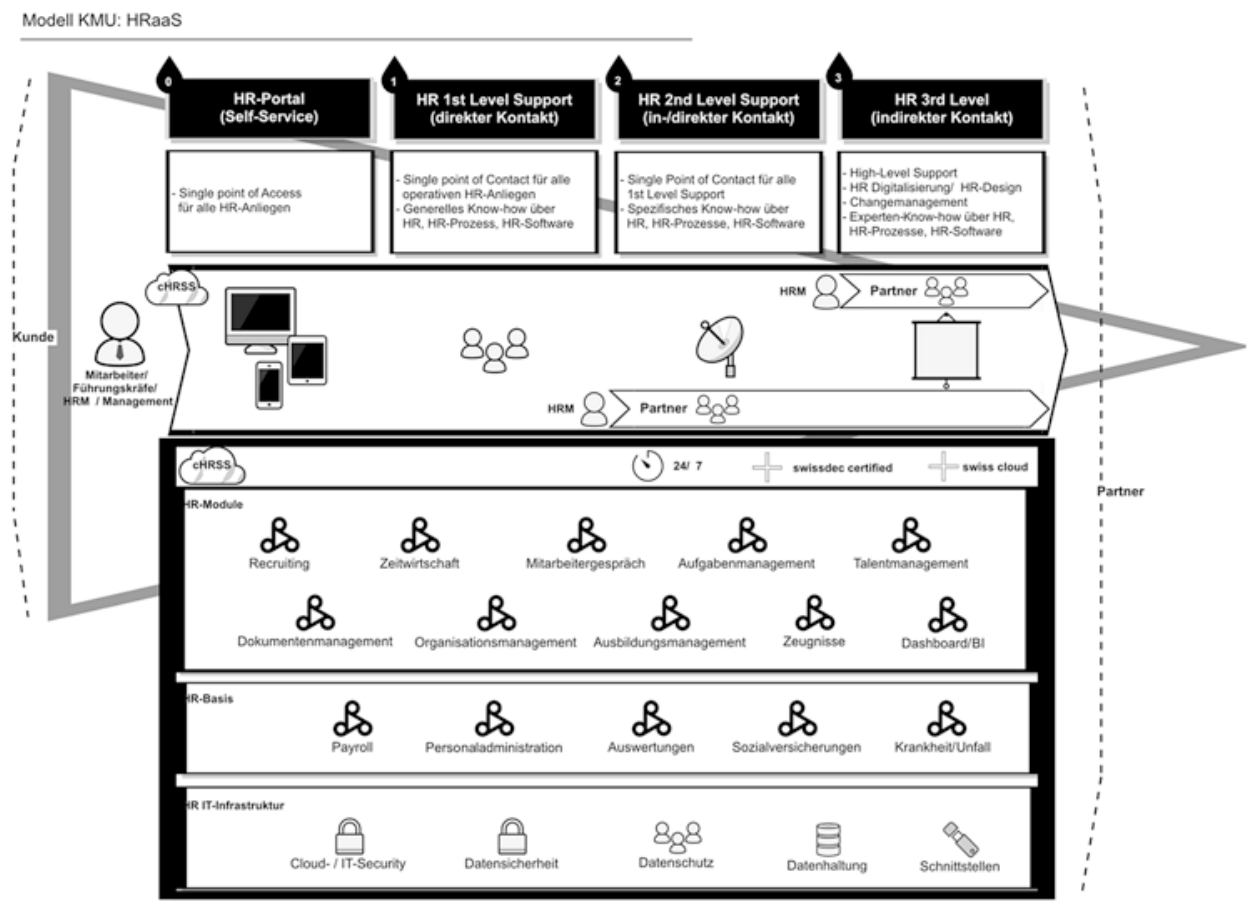

Abb. 10.4 Modifiziertes Modell für Human Resources as a Service (HRaaS). (Quelle: eigene Darstellung) 
terner Applikationen erlauben. Der Third Level Support wurde des Weiteren mit Blick auf den besonders seitens der KMU signalisierten Unterstützungsbedarf um das Supportfeld „Change Management“" ergänzt.

\subsection{Fazit und Ausblick}

Die Digitalisierung und die mit ihr einhergehende Digitale Transformation von Wirtschaft und Gesellschaft führen auch zu Änderungsbedarfen bei der Ausgestaltung des HRM in Unternehmen und Institutionen. In Folge werden zunehmend alle HR-Prozesse und -Tätigkeitsgebiete im Hinblick auf effizienz- und effektivitätssteigernde Digitalisierungsoptionen durch den Einsatz moderner Software- und Datenhaltungsmöglichkeiten geprüft. Vor allem im Bereich der administrativen, repetitiven Aufgabenerfüllung gibt es große Kostensenkungspotenziale für die Personalbereiche, insbesondere bei den KMU, die in Hinsicht auf den digitalen Reifegrad ihres HRM gegenüber den Großunternehmen noch einen Nachholbedarf haben. HR Shared Services bieten hier die Chance einer optimierenden Bündelung von relevanten Aktivitäten in einer zentralisierten Organisationseinheit, dem HR Shared Services Center, das intern oder extern angesiedelt sein kann und das durch einen hohen Automatisierungs- und Digitalisierungsgrad gekennzeichnet ist. In diesem Zusammenhang und in Verbindung mit neuen technologischen Entwicklungen werden skalierbare und hoch verfügbare Cloudlösungen als besonders zukunftsträchtig auch für HR-Anwendungen eingestuft. Die eigene Erhebung hat bestätigt, dass vor allem mittelgroße Unternehmen einen größeren Unterstützungs- und Entwicklungsbedarf bei der Digitalisierung ihres HRM aufweisen. Das auf diesen Unterstützungsbedarf hin ausgerichtete KMU-orientierte Modell „HR as a Service“ führt die Vorteile der HR-Digitalisierung von Shared Services und Cloud Computing in einem als Partner- und Geschäftsmodell interpretierbaren Servicekonzept zusammen. Das empirisch geprüfte und modular aufgebaute Servicemodell sieht für KMU einen abstufbaren cloudbasierten Bezug von HR-Dienstleistungen und -Software bei einem Partnerunternehmen vor, die mit einer großen Bandbreite flexibel genutzt werden können. Die KMU entscheiden jeweils für die einzelnen HRM-Aktivitäten über den gewünschten Externalisierungsgrad und können gestaffelt eine Digitalisierung ihrer HR-Prozesse voranbringen. Der Einsatz des HRaaSModells in Zusammenarbeit mit einem kompetenten Partner, der über die adäquate Infrastruktur, eine anwenderfreundliche HRM-Softwarelösung und eine sichere Datenhaltung in der Schweiz verfügt, hat ein großes Potenzial zur Effizienzsteigerung im HR-Bereich und ermöglicht den Unternehmen eine stärkere Konzentration auf das (HR-)Kerngeschäft.

Die digitale Transformation wird voraussichtlich die zukünftige Rolle des HRM deutlich verändern. Die weitgehende Digitalisierung der administrativen Prozesse und die Verwendung intelligenter HR-Softwarelösungen führen zur Eliminierung einer Vielzahl heutiger Aufgaben. Gleichzeitig werden Freiräume geschaffen, die eine neue Stufe der Professionalisierung des HRM ermöglichen. Der Personalbereich wird sich vor diesem Hintergrund noch stärker in Richtung eines strategischen Unternehmensentwicklungspart- 
ners entwickeln und die Geschäftsleitung bei komplexen Changeprojekten begleiten und unterstützen müssen. Losgelöst hiervon wird aber die heutige Kernaufgabe des HRM einer bedarfs- und zeitgerechten Bereitstellung von Personalressourcen resp. der mit ihnen verbundenen Kompetenzen und Fähigkeiten auch in Zukunft noch Fortbestand haben, auch wenn diese Aufgabe durch digitale Tools und HRaaS spürbar erleichtert werden kann.

\section{Literatur}

Abolhassan, F. (2016). Digitalisierung als Ziel - Cloud als Motor. In F. Abolhassan (Hrsg.), Was treibt die Digitalisierung? Warum an der Cloud kein Weg vorbeiführt (S. 15-26). Wiesbaden: Springer Gabler.

Altmeier, C. (2017). Steigerung des HR-Wertbeitrags durch Einführung von Shared Service Centern. In T. Eichenberg \& R. Bursy (Hrsg.), Management von internationalen HR Shared Service Centern. Implementierungsempfehlungen und Best Practice (S. 9-22). Wiesbaden: Springer Gabler.

Armutat, S., Bartholomäus, N., Franken, S., Herzig, V., \& Helbich, B. (Hrsg.). (2018). Personalmanagement in Zeiten von Demografie und Digitalisierung. Herausforderungen und Bewältigungsstrategien für den Mittelstand. Wiesbaden: Springer Gabler.

Bauer, W., \& Hofmann, J. (2018). Arbeit, IT und Digitalisierung. In J. Hofmann (Hrsg.), Arbeit 4.0 Digitalisierung, IT und Arbeit. IT als Treiber der digitalen Transformation (S. 1-16). Wiesbaden: Springer Vieweg (Edition HMD).

Blume, P. (2006). HR service delivery maturity model. In H. Kruppke, M. Gontard, \& M. Otto (Hrsg.), Human Capital Management. Personalprozesse erfolgreich managen (S. 67-85). Berlin/ Heidelberg: Springer.

Brühl, R., et al. (2017). Shared Services - Relevanz, Ziele und Entwicklungsstand. In T. M. Fischer \& M. Vollmer (Hrsg.), Erfolgreiche Führung von Shared Services (S. 3-23). Wiesbaden: Springer Gabler (ZfbF-Sonderheft, 70/17).

Clerc, I. (2018). Wissenschaftliches Arbeiten. Modul WAFT-Forschungstools Methoden-Werkzeuge (unveröffentlichtes Manuskript). Bern: Berner Fachhochschule.

Dressler, S. (2007). Shared Services, Business Process Outsourcing und Offshoring. Die moderne Ausgestaltung des Back Office - Wege zu Kostensenkung und mehr Effizienz im Unternehmen (1. Aufl.). Wiesbaden: Gabler.

Drupp, M. (2018). Gesundheitsförderung in der Arbeitswelt. Trends, Rahmenbedingungen und Beispiele guter Praxis unter besonderer Berücksichtigung der Unterstützungsmöglichkeiten durch die GKV. In A. Ternès \& C.-D. Wilke (Hrsg.), Agenda HR - Digitalisierung, Arbeit 4.0, New Leadership. Was Personalverantwortliche und Management jetzt nicht verpassen sollten (S. 67-85). Wiesbaden: Springer Gabler.

Fleischmann, A., Oppl, S., Schmidt, W., \& Stary, C. (2018). Ganzheitliche Digitalisierung von Prozessen. Perspektivenwechsel - Design Thinking - wertegeleitete Interaktion. Wiesbaden: Springer Vieweg.

Gleich, R., Knocke, H., \& Laux, T. (2017). SSC als Geschäftsmodell und Ansatzpunkte zur Geschäftsmodellinnovation. In T. M. Fischer \& M. Vollmer (Hrsg.), Erfolgreiche Führung von Shared Services (S. 149-166). Wiesbaden: Springer Gabler (ZfbF-Sonderheft, 70/17).

Greff, T., Gugler, K., \& Werth, D. (2018). Consulting 4.0 - Skalierbarkeit als 4 Gestaltungselemente der Digitalisierung für die Unternehmensberatung: Konzept, Ansätze und Kundenakzeptanz. In J. Hofmann (Hrsg.), Arbeit 4.0 - Digitalisierung, IT und Arbeit. IT als Treiber der digitalen Transformation (S. 53-78). Wiesbaden: Springer Vieweg (Edition HMD). 
Helfferich, C. (2011). Die Qualität qualitativer Daten. Manual für die Durchführung qualitativer Interviews (4. Aufl.). Wiesbaden: Springer Fachmedien.

Jakob, M. (2017). Entwicklung der HR SSC-Prozesse und Definition von Schnittstellen zu anderen HR-Funktionen. Unter Mitarbeit von Jakob. In T. Eichenberg \& R. Bursy (Hrsg.), Management von internationalen HR Shared Service Centern. Implementierungsempfehlungen und Best Practice (S. 121-137). Wiesbaden: Springer Gabler.

Kienbaum. (Hrsg.) (2015). Ergebnisbericht HR-Trendstudie 2015. Kienbaum Communications $\mathrm{GmbH} \&$ Co. KG. https://cdn-assets.kienbaum.com/downloads/HR-Trendstudie-Ergebnisbereicht-Kienbaum-Studie-2015.pdf?mtime=20160810112147. Zugegriffen am 02.03.2019.

KPMG. (Hrsg.) (2018). Cloud-Monitor 2018. Strategien für eine zukunftsorientierte Cloud Security und Cloud Compliance. http://hub.kpmg.de/cloud-monitor-2018?utm_campaign=Cloud\%20 Monitor\%202018\&utm_source $=$ aem\&_hstc $=214917896.71$ eddbd03ee28d721934f $2 \mathrm{a} 94 \mathrm{f} 75 \mathrm{f}$ 10c.1558128957377.1558128957377.1558128957377.1\&_hssc $=214917896.3 .155812895737$ 8\&_hsfp=3807373959. Zugegriffen am 12.04.2019.

Konrad, J., Schaltegger, Ch., \& Jansen, A. (2018). HR im digitalen Wandel. Veränderungen im Arbeitsalltag aus der Perspektive von HR-Fachleuten. Hrsg. v. HR Today. http://web.fhnw.ch/plattformen/blogs/wirtschaft/wp-content/uploads/sites/19/2018/10/FHNW-Studie-DigitalisierungKorr-V4.pdf. Zugegriffen am 29.04.2019.

Krcmar, H., Eckert, C., Roßnagel, A., Sunyaev, A., \& Wiesche, M. (Hrsg.). (2018). Management sicherer Cloud-Services. Entwicklung und Evaluation dynamischer Zertifikate. Wiesbaden: Springer Gabler.

Lamnek, S., \& Krell, C. (2016). Qualitative Sozialforschung. Mit Online-Materialien (6., überarb. Aufl.). Weinheim/Basel: Beltz.

Leyh, C., Bley, K., \& Ott, M. (2018). Chancen und Risiken der Digitalisierung - Befragungen ausgewählter KMU. In J. Hofmann (Hrsg.), Arbeit 4.0 - Digitalisierung, IT und Arbeit. IT als Treiber der digitalen Transformation (S. 29-51). Wiesbaden: Springer Vieweg (Edition HMD).

Mayring, P. (2016). Einführung in die qualitative Sozialforschung. Eine Anleitung zu qualitativem Denken (6., überarb. Aufl.). Weinheim/Basel: Beltz.

Mell, P., \& Grance, T. (2011). The NIST definition of cloud computing recommendations of the National Institute of Standards and Technology. NIST Special Publication 800-145. Gaithersburg: U.S. Department of Commerce.

Moll, L. E. (2012). Strategische Erfolgsfaktoren von Shared Services im Personalbereich. Eine praxisorientierte Analyse zur wertorientierten Unternehmensführung. Zugl.: Dortmund, Techn. Univ., Diss., 2011. Wiesbaden: Springer Gabler (Springer Gabler Research Mir-Edition).

Mund, C. (2017). Strategische Entscheidungen zur Fortführung oder zum Outsourcing eines internationalen HR Shared Service Centers. In T. Eichenberg \& R. Bursy (Hrsg.), Management von internationalen HR Shared Service Centern. Implementierungsempfehlungen und Best Practice (S. 287-302). Wiesbaden: Springer Gabler.

Peter, M. K. (2017). KMU-Transformation: Als KMU die Digitale Transformation erfolgreich umsetzen. Forschungsresultate und Praxisleitfaden. Olten: FHNW. https://kmu-transformation.ch/ wp/wp-content/uploads/2017/11/FHNW-HSW-Digitale-Transformation-KMU.pdf. Zugegriffen am 27.04.2019.

Reinnarth, J., Schuster, C., Möllendorf, J., Lutz, A., \& Buchenau, P. (2018). Chefsache Digitalisierung 4. 0 . Wiesbaden: Gabler.

Schneider, S., \& Sunyaev, A. (2015). Cloud-ServiceZertifizierung Ein Rahmenwerk und Kriterienkatalog zur Zertifizierung von Cloud-Services. Berlin/Heidelberg: Springer.

Scherer, E. K. (2017). Inhaltliche Ausgestaltung des Leistungsangebots für den Bereich Talent Management am Beispiel der E.ON Business Services GmbH. In T. Eichenberg \& R. Bursy (Hrsg.), 
Management von internationalen HR Shared Service Centern. Implementierungsempfehlungen und Best Practice (S. 203-219). Wiesbaden: Springer Gabler.

Sendler, U. (2018). Das Gespinst der Digitalisierung. Menschheit im Umbruch - auf dem Weg zu einer neuen Weltanschauung. Wiesbaden: Springer.

Swissdec. (2019). Was für Software-Hersteller wichtig ist. https://www.swissdec.ch/de/softwarehersteller. Zugegriffen am 17.04.2019.

Ternès, A. (2018). Digitale Transformation - HR vor enormen Herausforderungen. In A. Ternès \& C.-D. Wilke (Hrsg.), Agenda HR - Digitalisierung, Arbeit 4.0, New Leadership. Was Personalverantwortliche und Management jetzt nicht verpassen sollten. Wiesbaden: Springer Gabler.

Zöller, S. (2019). Ja zur Digitalisierung! Mit der richtigen Einstellung die Zukunftsfähigkeit des Unternehmens sichern. Wiesbaden: Springer Fachmedien.

Immanuel Zurbriggen Masterstudium in Betriebswirtschaft an der Berner Fachhochschule mit Vertiefung Corporate/Business Development und davor Bachelorstudium in Wirtschaftsinformatik an der Fachhochschule Nordwestschweiz. Mehrjährige Tätigkeit als Projektleiter und HR Business Analyst bei der Valiant Bank AG, verantwortlich für die Weiterentwicklung der HR-Softwares. Aktuell bei HR Service Center GmbH in der Geschäftsleitung und Führung des Geschäftsbereichs HR-Digitalisierung.

Prof. Dr. Jochen Schellinger ist Professor für Unternehmensführung und Organisation sowie Studiengangsleiter, Master of Science in Business Administration an der Berner Fachhochschule.

Open Access Dieses Kapitel wird unter der Creative Commons Namensnennung 4.0 International Lizenz (http://creativecommons.org/licenses/by/4.0/deed.de) veröffentlicht, welche die Nutzung, Vervielfältigung, Bearbeitung, Verbreitung und Wiedergabe in jeglichem Medium und Format erlaubt, sofern Sie den/die ursprünglichen Autor(en) und die Quelle ordnungsgemäß nennen, einen Link zur Creative Commons Lizenz beifügen und angeben, ob Änderungen vorgenommen wurden.

Die in diesem Kapitel enthaltenen Bilder und sonstiges Drittmaterial unterliegen ebenfalls der genannten Creative Commons Lizenz, sofern sich aus der Abbildungslegende nichts anderes ergibt. Sofern das betreffende Material nicht unter der genannten Creative Commons Lizenz steht und die betreffende Handlung nicht nach gesetzlichen Vorschriften erlaubt ist, ist für die oben aufgeführten Weiterverwendungen des Materials die Einwilligung des jeweiligen Rechteinhabers einzuholen.

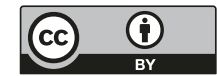

